\title{
gु
}

\section{Nuclear Binding Near a Quantum Phase Transition}

\author{
Serdar Elhatisari, ${ }^{1}$ Ning Li, ${ }^{2}$ Alexander Rokash, ${ }^{3}$ Jose Manuel Alarcón, ${ }^{1}$ Dechuan Du, ${ }^{2}$ Nico Klein, ${ }^{1}$ Bing-nan Lu, ${ }^{2}$ \\ Ulf-G. Meißner, ${ }^{1,2,4}$ Evgeny Epelbaum, ${ }^{3}$ Hermann Krebs, ${ }^{3}$ Timo A. Lähde, ${ }^{2}$ Dean Lee, ${ }^{5}$ and Gautam Rupak ${ }^{6}$ \\ ${ }^{1}$ Helmholtz-Institut für Strahlen- und Kernphysik and Bethe Center for Theoretical Physics, Universität Bonn, D-53115 Bonn, Germany \\ ${ }^{2}$ Institute for Advanced Simulation, Institut für Kernphysik, and Jülich Center for Hadron Physics, \\ Forschungszentrum Jülich, D-52425 Jülich, Germany \\ ${ }^{3}$ Institut für Theoretische Physik II, Ruhr-Universität Bochum, D-44870 Bochum, Germany \\ ${ }^{4}$ JARA-High Performance Computing, Forschungszentrum Jülich, D-52425 Jülich, Germany \\ ${ }^{5}$ Department of Physics, North Carolina State University, Raleigh, North Carolina 27695, USA \\ ${ }^{6}$ Department of Physics and Astronomy and $\mathrm{HPC}^{2}$ Center for Computational Sciences, \\ Mississippi State University, Mississippi State, Mississippi 39762, USA
}

(Received 21 March 2016; revised manuscript received 20 June 2016; published 19 September 2016)

\begin{abstract}
How do protons and neutrons bind to form nuclei? This is the central question of $a b$ initio nuclear structure theory. While the answer may seem as simple as the fact that nuclear forces are attractive, the full story is more complex and interesting. In this work we present numerical evidence from $a b$ initio lattice simulations showing that nature is near a quantum phase transition, a zero-temperature transition driven by quantum fluctuations. Using lattice effective field theory, we perform Monte Carlo simulations for systems with up to twenty nucleons. For even and equal numbers of protons and neutrons, we discover a first-order transition at zero temperature from a Bose-condensed gas of alpha particles ( ${ }^{4} \mathrm{He}$ nuclei) to a nuclear liquid. Whether one has an alpha-particle gas or nuclear liquid is determined by the strength of the alpha-alpha interactions, and we show that the alpha-alpha interactions depend on the strength and locality of the nucleon-nucleon interactions. This insight should be useful in improving calculations of nuclear structure and important astrophysical reactions involving alpha capture on nuclei. Our findings also provide a tool to probe the structure of alpha cluster states such as the Hoyle state responsible for the production of carbon in red giant stars and point to a connection between nuclear states and the universal physics of bosons at large scattering length.
\end{abstract}

DOI: 10.1103/PhysRevLett.117.132501

There have been significant recent advances in ab initio nuclear structure theory using a variety of different methods [1-7]. Much of the progress has been driven by computational advances, but we also have a better conceptual understanding of how nuclear forces impact nuclear structure. A key tool in making this connection is chiral effective field theory, which organizes the low-energy nuclear interactions of protons and neutrons according to powers of momenta and factors of the pion mass. The most important interactions are included at leading order (LO), the next largest contributions appear at next-toleading order (NLO), and then next-to-next-to-leading order (NNLO) and so on. See Ref. [8] for a recent review of the chiral effective field theory. While the progress in $a b$ initio nuclear theory has been impressive, there are gaps in our understanding of the connection between nuclear forces and nuclear structure. In this Letter, we discover an unexpected twist in the story of how nucleons selfassemble into nuclei. In order to make our calculations transparent and reproducible by others, we remove all nonessential complications from our discussion. For this purpose, we present lattice Monte Carlo simulation results using lattice interactions at LO in chiral effective field theory, together with Coulomb interactions between protons. In the lattice calculations discussed here we use a spatial lattice spacing of $1.97 \mathrm{fm}$ and time lattice spacing of $1.32 \mathrm{fm}$. We are using natural units where the reduced Planck constant $\hbar$ and the speed of light $c$ equal 1 .

Our starting point is two lattice interactions $A$ and $B$ at leading order in chiral effective field theory which are by design similar to each other and tuned to experimental lowenergy nucleon-nucleon scattering phase shifts. The details of these interactions and scattering phase shifts are presented in the Supplemental Material [9], but we note some important points here. The interactions appear at $\mathrm{LO}$ in chiral effective field theory and consist of short-range interactions as well as the potential energy due to the exchange of a pion. As the short-range interactions are not truly pointlike, they are actually what we call improved LO interactions. We write the nucleon-nucleon interactions as $V_{A}\left(\mathbf{r}^{\prime}, \mathbf{r}\right)$ and $V_{B}\left(\mathbf{r}^{\prime}, \mathbf{r}\right)$, where $\mathbf{r}$ is the spatial separation of the two incoming nucleons and $\mathbf{r}^{\prime}$ is the spatial separation of the two outgoing nucleons. The short-range interactions in $V_{A}\left(\mathbf{r}^{\prime}, \mathbf{r}\right)$ consist of nonlocal terms, which means that $\mathbf{r}^{\prime}$ and $\mathbf{r}$ are in general different. In contrast, the short-range interactions in $V_{B}\left(\mathbf{r}^{\prime}, \mathbf{r}\right)$ include nonlocal terms and also local terms where $\mathbf{r}^{\prime}$ and $\mathbf{r}$ are fixed to be equal. The main difference between interactions $A$ and $B$ is the degree of 
TABLE I. Ground state energies of ${ }^{3} \mathrm{H},{ }^{3} \mathrm{He},{ }^{4} \mathrm{He},{ }^{8} \mathrm{Be},{ }^{12} \mathrm{C},{ }^{16} \mathrm{O},{ }^{20} \mathrm{Ne}$ for interactions $A$ and $B$. We show LO results, $\mathrm{LO}+$ Coulomb results, and experimental data. All energies are in units of $\mathrm{MeV}$. The error bars denote one standard deviation errors.

\begin{tabular}{lcccrr}
\hline \hline Nucleus & $A(\mathrm{LO})$ & $B(\mathrm{LO})$ & $A(\mathrm{LO}+$ Coulomb $)$ & $B(\mathrm{LO}+$ Coulomb) & Experiment \\
\hline${ }^{3} \mathrm{H}$ & $-7.82(5)$ & $-7.78(12)$ & $-7.82(5)$ & $-7.78(12)$ & -8.482 \\
${ }^{3} \mathrm{He}$ & $-7.82(5)$ & $-7.78(12)$ & $-7.08(5)$ & $-7.09(12)$ & -7.718 \\
${ }^{4} \mathrm{He}$ & $-29.36(4)$ & $-29.19(6)$ & $-28.62(4)$ & $-28.45(6)$ & -28.296 \\
${ }^{8} \mathrm{Be}$ & $-58.61(14)$ & $-59.73(6)$ & $-56.51(14)$ & $-57.29(7)$ & -56.591 \\
${ }^{12} \mathrm{C}$ & $-88.2(3)$ & $-95.0(5)$ & $-84.0(3)$ & $-89.9(5)$ & -92.162 \\
${ }^{16} \mathrm{O}$ & $-117.5(6)$ & $-135.4(7)$ & $-110.5(6)$ & $-126.0(7)$ & -127.619 \\
${ }^{20} \mathrm{Ne}$ & $-148(1)$ & $-178(1)$ & $-137(1)$ & $-164(1)$ & -160.645 \\
\hline \hline
\end{tabular}

locality of the short-range interactions. Another difference is that there are extra parameters contained in interaction $B$, and these are used to reproduce $S$-wave scattering for two alpha particles.

We have used auxiliary-field Monte Carlo simulations to calculate nuclear ground state energies. In Table I we present the ground state energies of ${ }^{3} \mathrm{H},{ }^{3} \mathrm{He},{ }^{4} \mathrm{He},{ }^{8} \mathrm{Be},{ }^{12} \mathrm{C}$, ${ }^{16} \mathrm{O},{ }^{20} \mathrm{Ne}$ for interactions $A$ and $B$. While we use the notation meant for bound nuclei, in some cases the nuclear ground state is an unbound continuum state in our finite periodic box. We do not stabilize against decay to alpha particles. In fact, for the case of interaction $A$, all of the ground states in Table I are multialpha states. Details about the size of the box and the initial states used in the Monte Carlo simulations are provided in Supplemental Material [9]. The nuclei ${ }^{4} \mathrm{He},{ }^{8} \mathrm{Be},{ }^{12} \mathrm{C},{ }^{16} \mathrm{O},{ }^{20} \mathrm{Ne}$ are alphalike nuclei with even and equal numbers of protons and neutrons. We show results at leading order (LO) and leading order with Coulomb interactions between protons ( $\mathrm{LO}+$ Coulomb), as well as the comparison with experimental data. All energies are in units of $\mathrm{MeV}$. The lattice volume is taken large enough so that the finite-volume energy correction is less than $1 \%$ in relative error. The $\mathrm{LO}+$ Coulomb results for interaction $B$ are in good agreement with experimental results, better overall than the NNLO results in Ref. [3]. However, there is significant underbinding for interaction $A$ with increasing nucleon number. For interaction $A$, it is illuminating to compute the ratio of the LO energy for each of the alphalike nuclei to that of the alpha particle. For ${ }^{8} \mathrm{Be}$ the ratio is $1.997(6)$, for ${ }^{12} \mathrm{C}$ the ratio is $3.00(1)$, for ${ }^{16} \mathrm{O}$ it is $4.00(2)$, and for ${ }^{20} \mathrm{Ne}$ we have 5.03(3). These simple integer ratios indicate that the ground state for interaction $A$ in each case is a weakly interacting Bose gas of alpha particles. This interpretation is also confirmed by calculations of two-nucleon spatial correlations and local four-nucleon correlations.

To understand how interactions $A$ and $B$ can produce such completely different physics, we show their alphaalpha $S$-wave phase shifts in Fig. 1 . The LO results for interaction $A$ are shown with green triangles, $\mathrm{LO}+$ Coulomb results for $A$ are orange diamonds, LO results for $B$ are blue circles, and $\mathrm{LO}+$ Coulomb results for $B$ are red squares. The experimental data are shown with black asterisks [30]. The phase shifts are computed using auxiliary-field Monte Carlo simulations and a technique called the adiabatic projection method [31]. Interaction $B$ was tuned to the nucleon-nucleon phase shifts and the alpha-alpha $S$-wave phase shifts, and so the agreement with experimental data is very good. However, the phase shifts for interaction $A$ are small and even negative at larger energies. This would explain the large differences between interactions $A$ and $B$ for the energies of the larger alphalike nuclei in Table I.

What we have discovered is that alpha-alpha scattering is very sensitive to the degree of locality of the nucleonnucleon lattice interactions. In the Supplemental Material [9] we show that this dependence on the degree of locality is due to the compactness of the alpha-particle wave

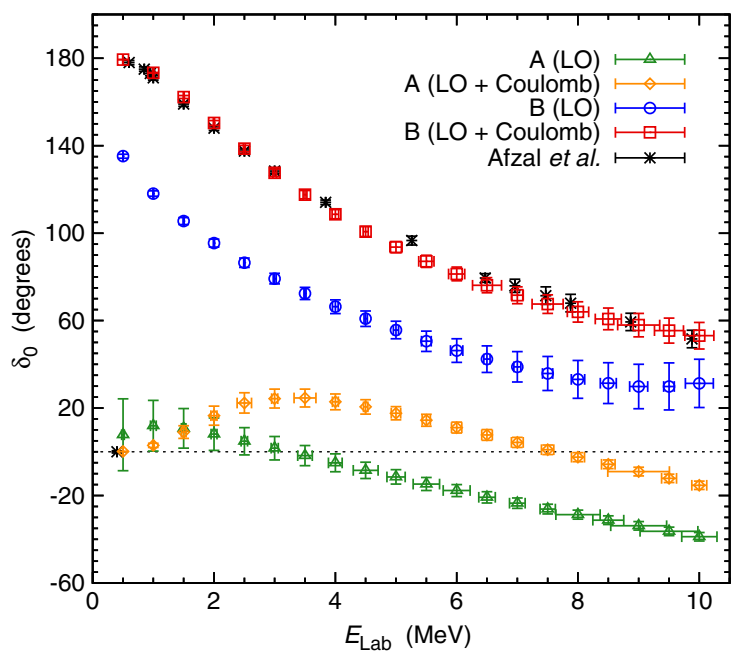

FIG. 1. Alpha-alpha $S$-wave scattering. We plot $S$-wave phase shifts $\delta_{0}$ for alpha-alpha scattering for interactions $A$ and $B$ versus laboratory energy. We show LO results for interaction $A$ (green triangles), $\mathrm{LO}+$ Coulomb for $A$ (orange diamonds), LO results for $B$ (blue circles), and $\mathrm{LO}+$ Coulomb results for $B$ (red squares). The phase shift analysis of experimental data are shown with black asterisks [30]. The theoretical error bars indicate one standard deviation uncertainty due to Monte Carlo errors and the extrapolation to infinite number of time steps. 
function. In contrast, the nucleon-nucleon scattering phase shifts make no constraint on the degree of locality of the nucleon-nucleon interactions. For example, if one starts with a purely local interaction, a unitary transformation can be used to define a new interaction which is highly nonlocal but having exactly the same phase shifts. The differences only become apparent in systems with more than two nucleons and can be understood as arising from three-body and higher-body interactions $[32,33]$. Interaction $A$ is a perfectly valid starting point for describing nucleonnucleon interactions. However, substantial higher-nucleon interactions will be needed to rectify the missing strength of the alpha-alpha interactions and the additional binding energy in nuclei.

The results we have found here suggest a strategy for improving future $a b$ initio nuclear structure and reaction calculations by incorporating low-energy light-nucleus scattering data in addition to nucleon-nucleon scattering data. This would be especially important for accurate calculations of key alpha capture reactions relevant to astrophysics such as alpha capture on ${ }^{12} \mathrm{C}$ [34]. One can view the extra step of fixing the degree of locality of the nucleon-nucleon interaction as preemptively reducing the importance of the required three-body and higher-body interactions. It is similar in spirit to other approaches that use nuclear structure and many-body observables to help determine the nucleon-nucleon interactions $[7,35,36]$.

Since alpha-alpha scattering is a difficult and computationally intensive $a b$ initio calculation, it is useful to discuss a simple qualitative picture of the alpha-alpha interaction in a tight-binding approximation. For any nucleon-nucleon interaction $V\left(\mathbf{r}^{\prime}, \mathbf{r}\right)$, we define the tight-binding potential $V_{\mathrm{TB}}(r)$ as the contribution that $V\left(\mathbf{r}^{\prime}, \mathbf{r}\right)$ makes to the effective interaction between alpha particles in the tightbinding approximation where the alpha particle radius $R_{\alpha}$ is treated as a small but nonvanishing length scale. In this simple approximation the interaction $V\left(\mathbf{r}^{\prime}, \mathbf{r}\right)$ contributes to the effective alpha-alpha interaction only in two possible ways. The first is what we call the direct term where $\left|\mathbf{r}^{\prime}-\mathbf{r}\right| \lesssim R_{\alpha}$, and the second is the exchange term where $\left|\mathbf{r}^{\prime}+\mathbf{r}\right| \lesssim R_{\alpha}$. All other terms are forbidden because the interaction is moving the nucleons to locations where there are no alpha particles. For the LO lattice interactions we consider here at lattice spacing $1.97 \mathrm{fm}$, we do not attempt to resolve the different microscopic mechanisms that can contribute to $V_{\mathrm{TB}}(r)$. However, calculations at smaller lattice spacings would find that the two-pion exchange interaction is responsible for a large attractive tight-binding potential at NNLO [8]. This observation connects well with the work of Ref. [37], which considered the role of the twopion exchange interaction in an effective field theory where alpha particles are treated as fundamental objects.

In Fig. 2 we show the tight-binding potential for the LO lattice interactions for $A$ and $B$. For our lattice calculations where space is discrete, we find that $R_{\alpha}$ is less than one

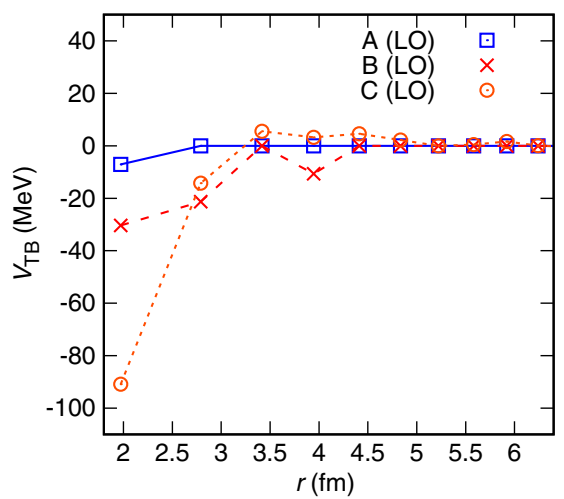

FIG. 2. Tight-binding potential. We plot the tight-binding potential versus radial distance for the LO interactions for $A, B$, and $C$, where $C$ is the interaction used in several previous lattice calculations [3,31]. Interaction $A$ is shown with blue squares and solid line, $B$ is drawn with red crosses and a dashed line, and $C$ is presented with orange circles and a short-dashed line.

lattice spacing and so the dependence on $R_{\alpha}$ drops out. We see that interaction $A$ has a very small tight-binding potential. This is consistent with the weak alpha-alpha $S$-wave interactions found in Fig. 1. In contrast, interaction $B$ has a stronger attractive tight-binding potential resulting from its short-range spin-isospin-independent local interaction. For comparison we also show in Fig. 2 the tightbinding potential for the leading-order interaction used in prior lattice calculations, which we call interaction $C[3,31]$.

In order to discuss the many-body limit, we switch off the Coulomb interactions and define a one-parameter family of interactions $V_{\lambda}=(1-\lambda) V_{A}+\lambda V_{B}$. While the properties of the two, three, and four nucleon systems vary only slightly with $\lambda$, the many-body ground state of $V_{\lambda}$ undergoes a quantum phase transition from a Bosecondensed gas to a nuclear liquid.

We sketch the zero-temperature phase diagram in Fig. 3. The phase transition occurs when the alpha-alpha $S$-wave scattering length $a_{\alpha \alpha}$ crosses zero, and the Bose gas collapses due to the attractive interactions [38,39]. At slightly larger $\lambda$, finite alphalike nuclei also become bound, starting with the largest nuclei first. The last alphalike nucleus to be bound is ${ }^{8} \mathrm{Be}$ at the so-called unitarity point where $\left|a_{\alpha \alpha}\right|=\infty$. Superimposed on the phase diagram, we have sketched the alphalike nuclear ground state energies $E_{A}$ for $A$ nucleons up to $A=20$ relative to the corresponding multialpha threshold $E_{\alpha} A / 4$. Empirically, we find that the quantum phase transition occurs at the point $\lambda_{\infty}=0.0(1)$. The uncertainty of \pm 0.1 is due to the energy levels having a slow dependence on $\lambda$ near $\lambda=0.0$. Since any $V_{\lambda}$ represents a seemingly reasonable starting point for the effective field theory at LO, one may end up crossing the phase transition when considering higher-order effects beyond LO. It is in this sense that we say nature is near a quantum phase transition. 


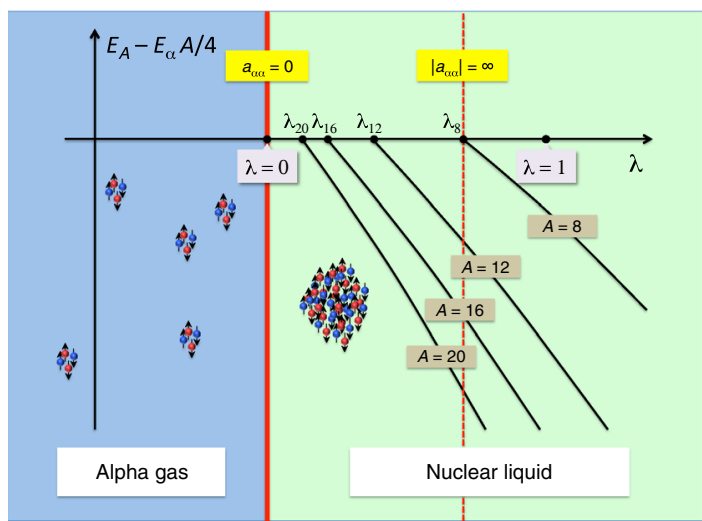

FIG. 3. Zero-temperature phase diagram. We show the zerotemperature phase diagram as a function of the parameter $\lambda$ in the interaction $V_{\lambda}=(1-\lambda) V_{A}+\lambda V_{B}$ without Coulomb included. The blue filled circles indicate neutrons, the red filled circles indicate protons, and the small arrows attached to the circles indicate spin direction. We show a first-order quantum phase transition from a Bose gas to nuclear liquid at the point where the scattering length $a_{\alpha \alpha}$ crosses zero. We have also plotted the alphalike nuclear ground state energies $E_{A}$ for $A$ nucleons up to $A=20$ relative to the corresponding multialpha threshold $E_{\alpha} A / 4$. The last alphalike nucleus to be bound is ${ }^{8} \mathrm{Be}$ at the unitarity point where $\left|a_{\alpha \alpha}\right|=\infty$.

The critical point for the binding of ${ }^{20} \mathrm{Ne}$ occurs at $\lambda_{20}=0.2(1)$. For the binding of the other alpha nuclei, we obtain $\lambda_{16}=0.2(1)$ for ${ }^{16} \mathrm{O}, \lambda_{12}=0.3(1)$ for ${ }^{12} \mathrm{C}$, and $\lambda_{8}=$ $0.7(1)$ for ${ }^{8} \mathrm{Be}$. One finds a sudden change in the nucleonnucleon density correlations at long distances as $\lambda$ crosses the critical point, going from a continuum state to a selfbound system. As $\lambda$ increases further beyond this critical value, the nucleus becomes more tightly bound, gradually losing its alpha cluster substructure and becoming more like a nuclear liquid droplet. The quantum phase transition at $\lambda_{\infty}=0.0(1)$ is the corresponding phenomenon in the many-body system, a first-order phase transition occurring for infinite matter.

By adjusting $\lambda$ in $a b$ initio calculations, we have a new tool for studying alpha cluster states such as the Hoyle state of ${ }^{12} \mathrm{C}$ and possible rotational excitations of the Hoyle state [40-45]. By tuning $\lambda$ to the unitarity point $\left|a_{\alpha \alpha}\right|=\infty$, we can continuously connect the Hoyle state wave function without Coulomb interactions to a universal Efimov trimer [46-48]. An Efimov trimer is one of an infinite tower of three-body states for bosons in the large scattering-length limit, with intriguing mathematical properties such as fractal-like discrete scale invariance. Another interesting system is the second $0^{+}$state of ${ }^{16} \mathrm{O}$ [49], which should be continuously connected to a universal Efimov tetramer [48,50,51]. This connection to Efimov states is now being investigated in followup work. The ability to tune the energies of alpha cluster states relative to alpha-separation thresholds provides a new window on wave functions and rotational excitations of alpha cluster states. By studying the $\lambda$ dependence of nuclear energy levels one can also identify the underlying cluster substructure. For example, the energy of a nuclear state which is a weakly bound collection of four alpha clusters will track closely with the four-alpha threshold $4 E_{4} \mathrm{He}_{\mathrm{e}}$ as a function of $\lambda$, while a state which is composed of ${ }^{12} \mathrm{C}$ and ${ }^{4} \mathrm{He}$ clusters will track more closely with $E_{{ }^{12} \mathrm{C}}+E^{4} \mathrm{He}$. Such an analysis may provide a new theoretical foundation for understanding clustering in nuclei and complement existing work on clustering in the literature [52-57], thereby strengthening the theoretical motivation for experimental searches of alpha cluster states in alphalike nuclei.

We acknowledge discussions with Hans-Werner Hammer, Thomas Luu, Lucas Platter, and Thomas Schäfer, and partial financial support from the Deutsche Forschungsgemeinschaft (Sino-German CRC 110), the Helmholtz Association (Contract No. VH-VI-417), BMBF (Grant No. 05P12PDFTE), the U.S. Department of Energy (DE-FG02-03ER41260), and U.S. National Science Foundation Grant No. PHY-1307453. Further support was provided by the EU HadronPhysics3 project, the ERC Project No. 259218 NUCLEAREFT, the Magnus Ehrnrooth Foundation of the Finnish Society of Sciences and Letters, MINECO (Spain), the ERDF (European Commission) Grant No. FPA201340483-P, and the Chinese Academy of Sciences (CAS) President's International Fellowship Initiative (PIFI) Grant No. 2015VMA076. The computational resources were provided by the Jülich Supercomputing Centre at Forschungszentrum Jülich and by RWTH Aachen.

[1] R. Roth, J. Langhammer, A. Calci, S. Binder, and P. Navratil, Phys. Rev. Lett. 107, 072501 (2011).

[2] H. Hergert, S. K. Bogner, S. Binder, A. Calci, J. Langhammer, R. Roth, and A. Schwenk, Phys. Rev. C 87, 034307 (2013).

[3] T. A. Lähde, E. Epelbaum, H. Krebs, D. Lee, U.-G. Meißner, and G. Rupak, Phys. Lett. B 732, 110 (2014).

[4] V. Soma, C. Barbieri, and T. Duguet, Phys. Rev. C 89, 024323 (2014).

[5] J. Carlson, S. Gandolfi, F. Pederiva, S. C. Pieper, R. Schiavilla, K. E. Schmidt, and R. B. Wiringa, Rev. Mod. Phys. 87, 1067 (2015).

[6] J. Simonis, K. Hebeler, J. D. Holt, J. Menendez, and A. Schwenk, Phys. Rev. C 93, 011302 (2016).

[7] G. Hagen et al., Nat. Phys. 12, 186 (2016).

[8] E. Epelbaum, H.-W. Hammer, and U.-G. Meißner, Rev. Mod. Phys. 81, 1773 (2009).

[9] See Supplemental Material at http://link.aps.org/ supplemental/10.1103/PhysRevLett.117.132501, which includes Refs. [10-29], for a discussion of the lattice interactions, scattering phase shifts, auxiliary field Monte Carlo simulations, adiabatic projection method, tight-binding approximation, and nuclear ground state energies. 
[10] E. Epelbaum, H. Krebs, D. Lee, and U.-G. Meißner, Eur. Phys. J. A 45, 335 (2010).

[11] N. Klein, D. Lee, W. Liu, and U.-G. Meißner, Phys. Lett. B 747, 511 (2015).

[12] J. Carlson, V. Pandharipande, and R. Wiringa, Nucl. Phys. A424, 47 (1984).

[13] B. Borasoy, E. Epelbaum, H. Krebs, D. Lee, and U.-G. Meißner, Eur. Phys. J. A 34, 185 (2007).

[14] B.-N. Lu, T. A. Lähde, D. Lee, and U.-G. Meißner, Phys. Lett. B 760, 309 (2016).

[15] V. G. J. Stoks, R. A. M. Klomp, M. C. M. Rentmeester, and J. J. de Swart, Phys. Rev. C 48, 792 (1993).

[16] E. Wigner, Phys. Rev. 51, 106 (1937).

[17] J.-W. Chen, D. Lee, and T. Schaefer, Phys. Rev. Lett. 93, 242302 (2004).

[18] D. Lee, Phys. Rev. Lett. 98, 182501 (2007).

[19] D. Lee, Prog. Part. Nucl. Phys. 63, 117 (2009).

[20] J. Hubbard, Phys. Rev. Lett. 3, 77 (1959).

[21] R. L. Stratonovich, Sov. Phys. Dokl. 2, 416 (1958).

[22] S. E. Koonin, J. Stat. Phys. 43, 985 (1986).

[23] J. Tjon, Phys. Lett. 56B, 217 (1975).

[24] A. Nogga, H. Kamada, and W. Glöckle, Phys. Rev. Lett. 85, 944 (2000).

[25] L. Platter, H.-W. Hammer, and U.-G. Meißner, Phys. Lett. B 607, 254 (2005).

[26] E. Epelbaum, H. Krebs, D. Lee, and U.-G. Meißner, Phys. Rev. Lett. 104, 142501 (2010).

[27] T. A. Lähde, T. Luu, D. Lee, U.-G. Meißner, E. Epelbaum, H. Krebs, and G. Rupak, Eur. Phys. J. A 51, 92 (2015).

[28] D. Lee, Phys. Rev. B 73, 115112 (2006).

[29] S. R. Beane, P. F. Bedaque, A. Parreno, and M. J. Savage, Phys. Lett. B 585, 106 (2004).

[30] S. A. Afzal, A. A. Z. Ahmad, and S. Ali, Rev. Mod. Phys. 41, 247 (1969).

[31] S. Elhatisari, D. Lee, G. Rupak, E. Epelbaum, H. Krebs, T. A. Lähde, T. Luu, and U.-G. Meißner, Nature (London) 528, 111 (2015).

[32] W. N. Polyzou and W. Glöckle, Few-Body Syst. 9, 97 (1990).

[33] S. K. Bogner, R. J. Furnstahl, and R. J. Perry, Phys. Rev. C 75, 061001 (2007).

[34] G. Imbriani, M. Limongi, L. Gialanella, F. Terrasi, O. Straniero, A. Chieffi, and O. A.d. Roma, Astrophys. J. 558, 903 (2001).
[35] P. Maris, J. P. Vary, and A. M. Shirokov, Phys. Rev. C 79, 014308 (2009).

[36] A. Ekström, G. R. Jansen, K. A. Wendt, G. Hagen, T. Papenbrock, B. D. Carlsson, C. Forssen, M. Hjorth-Jensen, P. Navratil, and W. Nazarewicz, Phys. Rev. C 91, 051301(R) (2015).

[37] E. Ruiz Arriola, arXiv:0709.4134.

[38] H. T. C. Stoof, Phys. Rev. A 49, 3824 (1994).

[39] Y. Kagan, A. E. Muryshev, and G. V. Shlyapnikov, Phys. Rev. Lett. 81, 933 (1998).

[40] Y. Funaki, A. Tohsaki, H. Horiuchi, P. Schuck, and G. Röpke, Phys. Rev. C 67, 051306 (2003).

[41] M. Chernykh, H. Feldmeier, T. Neff, P. von NeumannCosel, and A. Richter, Phys. Rev. Lett. 98, 032501 (2007).

[42] E. Epelbaum, H. Krebs, D. Lee, and U.-G. Meißner, Phys. Rev. Lett. 106, 192501 (2011).

[43] W. R. Zimmerman, N. E. Destefano, M. Freer, M. Gai, and F. D. Smit, Phys. Rev. C 84, 027304 (2011).

[44] E. Epelbaum, H. Krebs, T. A. Lähde, D. Lee, and U.-G. Meißner, Phys. Rev. Lett. 109, 252501 (2012).

[45] A. C. Dreyfuss, K. D. Launey, T. Dytrych, J. P. Draayer, and C. Bahri, Phys. Lett. B 727, 511 (2013).

[46] V. N. Efimov, Sov. J. Nucl. Phys. 12, 589 (1971).

[47] E. Braaten and H.-W. Hammer, Phys. Rep. 428, 259 (2006).

[48] T. Kraemer et al., Nature (London) 440, 315 (2006).

[49] E. Epelbaum, H. Krebs, T. A. Lähde, D. Lee, U.-G. Meißner, and G. Rupak, Phys. Rev. Lett. 112, 102501 (2014).

[50] H. W. Hammer and L. Platter, Eur. Phys. J. A 32, 113 (2007).

[51] J. von Stecher, J. P. D’Incao, and C. H. Greene, Nat. Phys. 5, 417 (2009).

[52] J. A. Wheeler, Phys. Rev. 52, 1083 (1937).

[53] D. M. Dennison, Phys. Rev. 96, 378 (1954).

[54] J. W. Clark and T.-P. Wang, Ann. Phys. (N.Y.) 40, 127 (1966).

[55] D. Robson, Phys. Rev. Lett. 42, 876 (1979).

[56] W. Bauhoff, H. Schultheis, and R. Schultheis, Phys. Rev. C 29, 1046 (1984).

[57] A. Tohsaki, H. Horiuchi, P. Schuck, and G. Röpke, Phys. Rev. Lett. 87, 192501 (2001). 